University of Wollongong

Research Online

Faculty of Engineering and Information

Faculty of Engineering and Information

Sciences - Papers: Part A

Sciences

$1-1-2016$

Student rules: exploring patterns of students' computer-efficacy and engagement with digital technologies in learning

Sarah Katherine Howard

University of Wollongong, sahoward@uow.edu.au

Jun Ma

University of Wollongong, jma@uow.edu.au

Jie Yang

University of Wollongong, jiey@uow.edu.au

Follow this and additional works at: https://ro.uow.edu.au/eispapers

Part of the Engineering Commons, and the Science and Technology Studies Commons

Research Online is the open access institutional repository for the University of Wollongong. For further information contact the UOW Library: research-pubs@uow.edu.au 


\title{
Student rules: exploring patterns of students' computer-efficacy and engagement with digital technologies in learning
}

\author{
Abstract \\ Teachers' beliefs about students' engagement in and knowledge of digital technologies will affect \\ technologically integrated learning designs. Over the past few decades, teachers have tended to feel that \\ students were confident and engaged users of digital technologies, but there is a growing body of \\ research challenging this assumption. Given this disparity, it is necessary to examine students' \\ confidence and engagement using digital technologies to understand how differences may affect \\ experiences in technologically integrated learning. However, the complexity of teaching and learning can \\ make it difficult to isolate and study multiple factors and their effects. This paper proposes the use of \\ data mining techniques to examine unique patterns among key factors of students' technology use and \\ experiences related to learning, as a way to inform teachers' practice and learning design. To do this, \\ association rules mining and fuzzy representations are used to analyze a large student questionnaire \\ dataset $(\mathrm{N}=8817)$. Results reveal substantially different patterns among school engagement and \\ computer-efficacy factors between students with positive and negative engagement with digital \\ technologies. Findings suggest implications for learning design and how teachers may attend to different \\ experiences in technologically integrated learning and future research in this area.
}

\section{Keywords}

technologies, engagement, efficacy, computer, students, patterns, exploring, rules, student, learning, digital

\section{Disciplines \\ Engineering | Science and Technology Studies}

\section{Publication Details}

Howard, S. K., Ma, J. \& Yang, J. (2016). Student rules: exploring patterns of students' computer-efficacy and engagement with digital technologies in learning. Computers and Education, 101 29-42. 


\title{
Student rules: Exploring patterns of students' computer-efficacy and engagement with digital technologies in learning
}

\begin{abstract}
1 Abstract
Teachers' beliefs about students' engagement in and knowledge of digital technologies will affect technologically integrated learning designs. Over the past few decades, teachers have tended to feel that students were confident and engaged users of digital technologies, but there is a growing body of research challenging this assumption. Given this disparity, it is necessary to examine students' confidence and engagement using digital technologies to understand how differences may affect experiences in technologically integrated learning. However, the complexity of teaching and learning can make it difficult to isolate and study multiple factors and their effects. This paper proposes the use of data mining techniques to examine unique patterns among key factors of students' technology use and experiences related to learning, as a way to inform teachers' practice and learning design. To do this, association rules mining and fuzzy representations are used to analyze a large student questionnaire dataset $(N=8,817)$. Results reveal substantially different patterns among school engagement and computer-efficacy factors between students with positive and negative engagement with digital technologies. Findings suggest implications for learning design and how teachers may attend to different experiences in technologically integrated learning and future research in this area.
\end{abstract}

Keywords: data mining, student beliefs, computer-efficacy, engagement, technology integration 


\section{Introduction}

Teachers' decisions about how to design learning, such as selecting teaching strategies, resources and assessments are, in part, mediated by what they think students will find engaging and how they believe students learn (Trigwell \& Prosser, 2004). In regard to technology integration, two guiding beliefs have been that students are confident users of and engaged in using digital technologies; and, technology use will increase engagement in learning and improve learning outcomes (e.g. Selwyn, 2009; Thompson, 2013). However, research has shown that many students are not confident or engaged in using digital technology (e.g. Margaryan, Littlejohn, \& Vojt, 2011; Wang, Hsu, Campbell, Coster, \& Longhurst, 2014; Warschauer \& Matuchniak, 2010). Disagreement on this point suggests a possible range of student experiences using technology, where some are engaged and others are not. It is important to understand variation in students' technology-related experiences, as misalignment between teacher and student expectations of technology use may lead to students' disengagement in learning. The purpose of this paper is to examine variations in students' confidence and engagement with digital technologies in learning and consider possible implications for teachers' learning design. A better understanding of these differences, and what they mean for learning, is needed to develop more effective and inclusive learning designs (Könings, Seidel, \& van Merriënboer, 2014b; Li, 2007; Skryabin, Zhang, Liu, \& Zhang, 2015).

To do this, we first address teachers' perceptions of students' needs and experiences in the classroom, followed by a conceptual framework of key factors affecting students use of information and communication technologies (ICTs) in learning. Data mining techniques, association rules mining and fuzzy representation, are used in the analytic framework. Data mining techniques can provide new insight into relations among known factors of digital 
integration, which can build on existing knowledge (Baker, 2010). Our analysis broadly examines eight key factors of ICT use, and then focuses on ICT engagement, computer-efficacy and school engagement. Results show two distinctly different patterns among these factors, which suggest differences in students' experiences in technologically integrated learning. Implications for learning design and student support when using ICTs are discussed, as well as directions for future research and model development.

\section{Teachers' perceptions of students' needs}

In education, there is still a strong belief that young people are able to confidently use digital technologies, and that they want to use these tools in learning. This belief has influenced how public and educational systems think about technology integration and learning (Margaryan, Littlejohn, \& Vojt, 2011; Selwyn, 2009; Thompson, 2013). It also affects how teachers select and integrate digital technologies in the classroom. However, assumptions about students' knowledge of and engagement in digital technologies can be problematic in teachers' learning designs (Philip \& Garcia, 2013).

Often teachers will be motivated to select and integrate digital technologies that are perceived to be of value. In part, this value is ascribed by how much they feel it may engage students and support learning (Ertmer \& Ottenbreit-Leftwich, 2010; Inan \& Lowther, 2010; Kim, Kim, Lee, Spector, \& DeMeester, 2013; Mumtaz, 2000). For example, Ottenbreit-Leftwich et al.'s (2010) study of teachers' perceptions of digital technologies found that teachers largely believed "technology could be used to engage and motivate students" (p. 1331). Bebell and Kay (2010) found that $83 \%$ of middle school teachers in a large-scale 1-to-1 laptop program in the United States felt traditional students' engagement in learning improved when using laptops. 
Further, $84 \%$ of teachers felt low achieving students were more engaged and $71 \%$ felt high achieving students were more engaged.

While there is a large amount of research looking at students' use of digital technologies, further research is needed to understand their "reactions or even behaviors during learning procedures which may 'engage' them," particularly in relation to educational practices (Pellas, 2014, p. 159). In studies such as Bebell and Kay's (2010), student engagement in digital technologies has often been reported by teachers or a researcher observes it, rather than by students. However, perceptions of what students need in learning should relate to their experiences, beliefs and knowledge (Hughes, 2005; Koehler et al., 2007; Köning et al., 2014). If differences between teachers and students' expectations of how learning happens and what is being learned are too large, students will be at risk of becoming unengaged and may struggle to develop learning and thinking skills (Vermunt \& Verloop, 1999).

Teachers have a high level of accuracy when judging students' academic achievement (e.g. Südkamp, Kaiser \& Möller, 2012), but research has shown a disconnect between teacher and student experiences in and perceptions of learning (e.g. Könings et al., 2014; Perrotta, 2013). Könings et al.'s (2014) found that only $30 \%$ of teachers and students were in agreement about expectations of learning and what was being learned in the classroom. Moreover, not all students report the same experience or quality of learning (Ellis, Goodyear, Bluic \& Ellis, 2011). The benefits of using digital technologies in the classroom come from teaching rather than the technology (Tamin, Bernard, Borokhovski, Abrami, \& Schmid, 2011; Warschauer et al., 2014), so how teachers understand students' capability and experience in learning is critical for technology integration to be effective. 
Therefore, while teachers may feel students like to use digital technologies, basing learning design on this assumption may be risky. To effectively integrate digital technologies in learning, it is important to understanding the possible breadth of student experience using digital technologies, and question if and how digital technologies are engaging for students.

\section{Factors affecting students' use and experiences}

Digital technologies used in learning include, but are not exclusively, the use of laptops, smartphones and tablets, various software packages, online resources, etc. (e.g. Inan \& Lowther, 2010; Thompson, 2013). In regard to young people's actual use of digital technologies, research has shown that it is generally low-level (Margaryan et al., 2011; Wang et al., 2014). Personal interests and entertainment dominate use, and as a result, young people are not necessarily confident or engaged with using digital technologies to learn (Warschauer, Zheng, Niiya, Cotten, \& Farkas, 2014). In regard to learning, most students have not used digital technologies in deep and/or critical ways (e.g. Thompson, 2013; Wang, Hsu, Campbell, Coster, \& Longhurst, 2014; Waycott, Bennett, Kennedy, Dalgarno, \& Gray, 2010). However, this finding is not necessarily consistent across all young people. Students from higher socio-economic backgrounds, who also often have higher levels of access, are more likely to have experienced using technology to support critical thinking than students of lower socio-economic background (Perrotta, 2013; Warschauer \& Matuchniak, 2010). Access to digital technologies and how this translates to use of and engagement with different tools is complex (e.g. Bennett \& Maton, 2010). However, some studies have found positive effects on engagement in school and learning from increased access to technology in school (e.g. Bebell \& Kay, 2010) and increased use outside school (e.g. Wang et al., 2014). Yet, research has also shown that young people who frequently use technology outside of school do not feel it is necessary in learning (e.g. Glušac, Makitan, 
Karuović, Radosav, \& Milanov, 2015). Conflicting findings reaffirm that young people do not all have the same experiences or feelings about using digital technologies (Bennett \& Maton, 2010; Hinostroza, Matamala, Labbé, Claro, \& Cabello, 2014). This suggests they will experience technologically integrated learning differently (Hatlevik, Guðmundsdóttir, \& Loi, 2014; Moos \& Azevedo, 2009).

More evidence is needed to understand variations in students' engagement with digital technologies, and how this may relate to learning and integrated learning design (Pellas, 2014; Tamim et al., 2011). Research has identified a number of key factors that could be examined to explore variation in students' experiences using digital technologies in learning, such as students' confidence using technology, beliefs about learning, engagement in school and technology, which all have an effect on experiences technology use and learning.

\subsection{ICT engagement}

Student engagement can be understood as the cognitive process, active participation and emotional involvement in a learning procedure (Pellas, 2014). ICT engagement specifically addresses involvement and participation in using digital technologies (Christoph et al., 2015). Teachers have tended to value ICTs they feel will engage students in learning as a method of increasing active participation in learning (Baylor \& Ritchie, 2002; Ottenbreit-Leftwich, Glazewski, Newby, \& Ertmer, 2010).

\subsection{Computer-efficacy}

Computer-efficacy relates to positive experiences and confidence using digital technologies, which shapes how individuals feel about their ability to perform future computerrelated tasks (Compeau \& Higgins, 1995). Higher levels of computer-efficacy have been related to higher ICT engagement (Laird \& Koh, 2005). Students' beliefs about their ability to use 
digital technologies are an important factor in how they will engage in technologically integrated learning (Hatlevik, Guðmundsdóttir, \& Loi, 2014; Moos \& Azevedo, 2009; Tzeng, 2009). However, using digital technologies in learning requires students to be able to perform a range of computer-related tasks. Harris, Mishra and Koehler (2009) suggest that activities should be identified to better understand how digital technologies relate to learning. Computer-related learning activities can be divided into three types, based on increasing complexity of the task. The first type is productivity tasks that facilitate learning, which includes basic email communication, simple online searching and editing a word processing document. These are very common in the classroom (e.g. Bebell \& O’Dwyer, 2010). The second type is processing information and data, such as creating charts, using simulations or discerning quality of information, which are more likely to relate to higher order thinking skills (e.g. Kozma, 2003). The third type is creating tasks, such as multimedia objects and websites, where students are creating products to demonstrate understanding and learning (e.g. Bebell \& Kay, 2010). Research has shown that students are often adept at productivity tasks, such as simple communication and online searching, but less familiar with more sophisticated tasks, such as those relating to processing and creating (e.g. Thompson, 2013; Wang et al., 2014).

\subsection{Learning preferences}

Teachers' decisions about how to teach will be guided by how they think students will learn (Trigwell \& Prosser, 2004). Research has identified that self-paced and collaborative learning strategies, such as students collaboratively creating complex products (e.g. collaborative writing) and exploring ideas on their own (e.g. working with data), are most effectively supported by digital technologies (Ertmer et al. 2012). However, these approaches to learning may not be preferred by students, which would have implications for engagement in and learning 
through certain types of learning designs (Kennedy et al. 2008; Margaryan et al., 2011; Webb \& Cox, 2004).

\subsection{Learning beliefs}

Extending the concept of learning preferences, students will hold certain beliefs about thinking, ways of knowing and how knowledge is constructed (Hofer \& Pintrich, 1997). These conceptions will influence beliefs about learning. For example, some students may feel they learn better through investigating problems on their own, while others may feel working in a group is better for learning. Teachers may hold certain beliefs about how students should learn, which may or may not be shared by students (Könings et al., 2014). These may include aspects of how they learn through a range of different task types and learning designs. However, students' beliefs can be changed through learning design and teacher modeling of new approaches to learning (Muis \& Duffy, 2013).

\subsection{ICT learning beliefs}

Research has shown that digital technologies most effectively support learning through critical engagement with content, development of cognitive skills and authentic learning tasks relating to students' own experiences (Lowther et al., 2012, Ottenbreit-Leftwich et al., 2010; Wang et al., 2014). However, students may not believe digital technologies are necessary in their learning; and, they would therefore be less engaged in their use (e.g. Margaryan et al., 2011). While students will hold specific beliefs about how well they learn with ICTs, these beliefs can be affected by teachers' beliefs about and use of ICTs (Gibson et al., 2014).

\subsection{School engagement}

Students engaged in school and learning will be attentive, exhibit interest and actively participate in learning (Reyes, Brackett, Rivers, White \& Slovey, 2012). Students who are 
engaged in learning are more likely to do well in school. Research has argued that access to digital technologies improves engagement in learning (e.g. Bebell \& Kay, 2010) and that there is an association between technology affinity and positive attitudes towards school (e.g. Mills et al., 2013). Further, it has also be argued that without use of digital technologies students may be less satisfied with and engaged in school (e.g. Kolikant, 2012).

\subsection{Teacher directed ICT use}

Students' beliefs about the value of ICTs in learning are affected by values of their teachers (Gibson et al., 2014). Research has attributed variations in students' learning to the types of technology-related tasks students are asked to perform in the classroom (Fairlie \& London, 2011; Lei \& Zhao, 2007; Skryabin et al., 2015). More complex tasks, such as creating websites, multimedia resources and programming, can have a positive impact on learning (Lei \& Zhoa, 2007). It is thought that responsibility of introducing complex uses of digital technologies falls with the teacher (Thompson, 2013).

\subsection{ICT importance in subject areas}

Teachers' attitudes about digital technologies differ among subject areas (Hennessy et al., 2003; Author/s, 2015), which affects how students value technology in learning (Gibson et al., 2014; Roehrig et al., 2007). The value of technologies in subject areas, as well as the types of tasks performed in different subject areas provides important information about the underlying principles of teaching and learning in that discipline (Author/s, 2011).

The above key factors of students' digital technology use in learning provide a framework through which different experiences can be explored. While factors presented here have been previously explored educational technology research, using a data mining approach 
can facilitate new analysis and possible identification of unique patterns to extend existing knowledge and inform learning.

\section{Conceptual framework}

\subsection{Data mining approach}

"Data mining is the process of automatically discovering useful information in large data repositories" (Tan, Steinbach \& Kumar, 2005). Data mining is an inductive process, which makes it different from more traditional statistical approaches, which seek to fit data to a hypothesized model or fit new data to an existing validated model (Brieman, 2001). The inductive process is referred to as "knowledge discovery" and does not assume a particular model. Rather, the aim of knowledge discovery is to identify unique patterns and trends in data (Baker, 2010). Results can then be visualized as a graph to create a model.

Educational data mining (EDM) is an emerging field, which aims to develop methods of exploring data and discovering meaningful patterns from educational settings (Baker \& Yacef, 2009; Levy \& Wilensky, 2011). EDM seeks to understand educational data from "far outside the purview of what data were originally intended to study" (Baker, 2010, p. 113). It encompasses a range of statistical approaches, traditional and from data mining, to support relationship analysis, prediction, clustering, discovering or improving domain knowledge structures, refine educational theories and provide learning support (Baker \& Yacef, 2009).

The current research does not aim to create new methods, but to apply well-proven data mining approaches, in particular association rule mining and fuzzy representation, to answer educational questions. Association rule mining is the primary method for relationship analysis and is used to identify interesting relationships in a dataset. These relationships are expressed in the form of association rules: 


\section{$A \rightarrow C$}

The rule contains an antecedent $(\mathrm{A})$ part and a consequence $(\mathrm{C})$ part. The logic of the rule is the likelihood of "IF teachers use a laptop frequently (A), then teachers will direct more ICT use in classroom learning $(\mathrm{C}) . "$ Each part of a rule may contain multiple antecedents and consequences, such as "IF $\mathrm{A}_{1}$ and $\mathrm{A}_{2}$, then $\mathrm{C}_{1}$ and $\mathrm{C}_{2}$." Analysis of a dataset can result in thousands of rules, some of which are more significant than others. The three most common critical measurements determining the significance of rules are support degree, confidence degree and lift (Tan, Kuman \& Srivastava, 2004). Support degree indicates to what extents both the antecedent(s) and consequence(s) occur simultaneously in the dataset. Confidence degree indicates to what extents the consequence(s) occurs following the antecedent(s). Lift measures correlation between the antecedent(s) and consequence(s). Lift can be used to estimate prediction performance. Detailed definitions of these measurements can be found in Han, Kamber and Pei (2012).

Fuzzy representation techniques aim to describe concepts and perceptions using fuzzy set theory. Fuzzy sets are widely used in decision analysis, approximate reasoning, engineering, pattern recognition and information systems (Klir \& Yuan, 1995). It is used to handle values or concepts have unclear semantic boundaries or semantic overlap (Chen \& Weng, 2009).

Questionnaires often contain responses that may be fuzzy (e.g. multiple-response questions and Likert-type scales) while others are crisp (e.g. numeric rankings and discrete categories). Fuzzy representation provides a way to descript fuzzy responses. For example, we can express the fuzzy response "frequent ICT user in teaching" as:

$$
\text { frequent user }(h)=\left\{\begin{array}{ll}
\frac{h}{3}, & h<3 \\
1, & h \geq 3
\end{array}\right. \text {, }
$$


where $h$ represents how many hours the user uses ICT in teaching per day. The fuzzy response can be represented in a graph (see Figure 1).

$<<$ insert Figure 1 here $>>$

It can be claimed that a user who spends one hour daily on using ICT will be treated as a "less frequent" user. The more hours a user spends on ICT use the more frequent user. Experts are needed to review the definitions of fuzzy representation to insure they are meaningful. Fuzzy representations can then be used as categories and included in association rules mining. Using fuzzy representation, it is possible to standardize (similar to calculating z-scores) different types of data for use in association rule mining, by extracting and standardizing semantic information embedded within responses. Creation of fuzzy representation can also increase sensitivity to variation among participants' responses. An example of this is the use of the standard Likert-type item of "strongly agree," "agree," "disagree" and "strongly disagree." These questions do not contain a true scale and will be interpreted differently by participants; therefore, using fuzzy representation can better describe the potential uncertainty and overlap of those responses. In this study, fuzzy representations are calculated for each of the key factors presented.

\subsection{Purpose of the study}

The aim of the current analysis is to examine variations in students' confidence and engagement with digital technologies in learning and consider possible implications for teachers' learning design. The specific research question for this investigation was: What are different patterns occurring among key factors relating to students' experiences in technology integration? 


\section{Method}

Data from a large-scale study of the Australian Digital Education Revolution in New South Wales (DER-NSW) was used to explore students' perceptions of technology integration. The DER was a federally funded program aiming to provide all secondary (Years 9-12) students and teachers across Australia with ICTs (Department of Education Employment and Workplace Relations [DEEWR], 2012). Across the country, each state engaged with the program differently. In NSW, a one-to-one laptop program was implemented. Through this program, all full-time secondary-level teachers and Year 9 students were provided with Lenovo laptops between 2009 and 2013. Students kept the laptops until they completed high school.

The DER-NSW one-to-one laptop program was evaluated over four years (2010-2013) through online questionnaires and school cases studies. This design allowed for collection of broad descriptive baseline data, which guided a more detailed exploration of the program through case studies. A full description of the study can be found in Author/s (2013) final report.

\subsection{Participants}

The DER-NSW study included all government secondary schools across the state $(N=$ 436). In 2010, all secondary teachers and Year 9 students were invited to participate.

Participation in the study was voluntary and there was no control group. These cohorts were resurveyed in 2011 and 2012. For comparison, in 2012 a second Year 9 cohort was invited to participate and sampled again in 2013 as Year 10 students. In 2012, of the approximately 50,000 Year 9 students in NSW government schools, 21,795 (44\%) students completed one of two questionnaires, which were assigned randomly by school. Questionnaire Part A, completed by 12,978 students, focused on students' use of digital technologies. Part B focused on learning with technology and was completed by 8,817 students. Part A and B were triangulated on several 
measures, so students only completed one version. Responses to Part B of the questionnaire were included in the current analysis to explore students' learning experiences with technology.

\subsection{Data collection instrument}

The student questionnaire Part B covered five main subscales: School Engagement, Computer Use, Your Learning, Your Subjects and Your Intentions. The School Engagement subscale is from the NSW DEC Student School Life Survey (SPL-SSL), which provides the department with student feedback on schools. This scale was adapted from the nationally validated School Life Questionnaire (Australian Council for Educational Research, 2009). The Computer Use subscale was adapted from the Program for International Student Assessment (PISA) ICT use and familiarity measure (OECD, 2006). It included items on frequency of use and confidence (computer-efficacy) performing computer tasks, such as Internet searches (Productivity), data manipulation (Processing) and creating a web page (Creating). Subscale Your Learning addressed students' learning preferences, drawn from the New South Wales SchoolMap Best Practices Statements (Department of Education and Training, 2002). Your Subjects asked students to consider bases of achievement and success in different subject areas (Lamont \& Maton, 2010). Your Intentions included standard department questions regarding students' intentions to leave school early, begin to work or enroll in study after graduation. A draft of the questionnaire was pilot tested in 2009 at three schools and revised. The final questionnaire contained 147 items, grouped into 31 measures. Reliability of the final questionnaire was determined to be high $($ alpha $=.99)$ and for each of the main subscales (alpha $=.81$ to $.95 ;$ see Appendix A). 


\subsection{Analysis}

Data mining analysis consisted of three main modules: factor generation, creating fuzzy representations and association rules mining. In the first module, factor generation was conducted by identifying key questions from the questionnaire data and eliminating irrelevant or redundant items. This module required domain knowledge to extract relevant questionnaire items, thereby focusing on particular pattern discovering in the analysis. The following eight main factors, covering 16 sub-factors, were identified: Computer-Efficacy ( 3 sub-factors), ICT Engagement (3 sub-factors), Learning Preferences (3 sub-factors), Learning Beliefs (3 subfactors), ICT and Learning Performance (1 sub-factor), School Engagement (1 sub-factor), Teacher Directed ICT Use Frequency (1 sub-factor) and ICT Importance in Subject Areas (1 sub-factor; see Table 1).

<insert Table 1 here>>

To explore the eight main factors, descriptive statistics were calculated, correlation and regression analysis were conducted.

In the second module, original data was pre-processed for analysis by rescoring raw data into computable variables to create fuzzy representations. To do this, a three-step algorithm was employed. The first step removed all missing responses from the eight factors in Table 1. In the second step, depending on the factor, scores were aggregated (e.g. averaged, sums, etc.). In the last step, final scores were converted into discrete values using sets of fuzzy representations. Each of the fuzzy concepts was labeled by an understandable linguistic term, such as positive and higher.

In the final mining module, association rules analysis was conducted on extracted factors to identify where potential significant relationships may exist. To do this, the dataset was split 
based on responses to the ICT Engagement factor. Students' engagement with ICT was identified as a motivating factor in teachers' use of digital technologies (Ottenbreit-Leftwich et al., 2010). Therefore, this was chosen as a starting point to investigate students' experiences in technologically integrated learning. Two datasets were created. Dataset 1 included only students reporting positive ICT Engagement. Dataset 2 included students reporting negative ICT Engagement. The apriori algorithm in R package was used for the analysis, in which the minimum support degree was set to 0.3 , minimum confidence degree at 0.7 , and the lift measure greater than 1.0.

Graph representations were used to visualize and interpret results of association rules mining. More precisely, rules from the two datasets were converted to a directed graph, in which each factor from the antecedent and consequence set was associated with a node in the graph. A directed connection is made between two factor nodes if factors exist in the same association rule. For instance, as discussed earlier, a typical rule takes the form $A \rightarrow C$, where A and $\mathrm{C}$ represent the antecedent and consequent of the rule, respectively. A directed edge (in the form of an arrow) is used to connect node $\mathrm{A}$ to $\mathrm{C}$, which implies that the factor A has a high probability of being associated with the factor $\mathrm{C}$.

This analysis focuses on patterns of connectivity among the factors, such as which factors are important and how they group together. There are two important aspects of these patterns. The first is centrality. An important factor that is likely to be associated with many other factors will have a high degree centrality (Stephenson \& Zelen, 1989). Secondly, how factors cluster together is important. Clustering represents groups of factors that are associated with, and located near, each other in a graph, which informs how they may influence each other (Newman, 2003). Clustering is based on the frequency of associations among groups of nodes, 
also referred to as density. In this exploratory analysis, we have used soft clustering, so nodes may belong to more than one cluster (Yu, Yu \& Tresp, 2005).

\section{Results}

The students participating in the Part B questionnaire represented 216 secondary schools from across the state. They were evenly divided between male (49\%) and female (51\%). Of this sample, only $8.5 \%$ identified as being of either or both Aboriginal and Torres Strait Islander. Both of these distributions were representative of the wider school population (Australian Bureau of Statistics, 2016). The majority of students reported having a computer at home (96\%) and that the computer was connected to the Internet (93\%).

Normality of the dataset is not an assumption in data mining. However, given the size of the dataset, it can be considered normal for correlation analysis and regression (Amemiya \& Anderson, 1990). A full report of students' responses to all items in the eight key factors has been provided in Supplementary Table A. Descriptive statistics for the eight main aggregated factors are presented in Table 2.

$<<$ Insert Table 2 here $>>$

Results from Table 2 show that students, as a whole, reported weak engagement in school. Students reported positive beliefs about learning and learning preferences (see Table 1 for question types). They reported weak engagement with ICTs, and they felt they were able to perform most computer-related tasks without help. They did not feel ICT was important in subject areas; yet, they felt it helped their learning. Correlation analysis confirmed significant weak to medium strength relationships among all factors $(r=.06$ to .46$)$. 
As an additional exploratory analysis, and to consider the factors as a whole model, multiple linear regression was conducted. Treating ICT Engagement as the dependent variable and using the enter method, it was found that the other seven factors explained $17 \%$ of variance in students' ICT Engagement, $F(7,6469)=182.80, p<.01, R^{2}=.17, R_{\text {Adjusted }}^{2}=.16$. Factors were predictive of ICT Engagement $(p<.01)$, except Learning Preferences $(p=.07)$. The factor was retained in the model for its theoretical importance and strong relationship with School Engagement and ICT Learning Performance.

Having confirmed that relationships existed among all eight factors, data mining techniques were then used to explore the two ICT engagement datasets for unique patterns. Table 3 presents the summary of total association rules extracted from each.

$<<$ Insert Table 3>>

The averaged support degree for rules from both datasets averaged .39, indicating that most rules included less than $40 \%$ of the participants. However, the measurement of confidence in rules was high, averaging $91 \%$. The high degree of confidence suggests that patterns were strong within the datasets. A higher lift is observed for positive attitudes on ICT Engagement (Dataset 1), which suggest this group is quite different from the whole group and the negative sample (Dataset 2).

Next, the proportion of factors appearing in rules for each dataset was calculated (e.g. how many times School Engagement appears as an antecedent or consequent in Dataset 1; see Table 4). This step identified which were the most important factors and patterns in relation to ICT engagement.

$<<$ Insert Table 4>. 
The largest proportion of rules contained factors of Learning Beliefs and Learning Preferences. This suggests strong relationships among Learning Beliefs, Learning Preferences and ICT Engagement. However, the frequency of rules was similar across both datasets, suggesting these factors were not strongly affected by variations in positive or negative ICT engagement. The largest differences in rule proportions were observed in Computer-Efficacy and School Engagement. Results show an overall 7\% difference in the proportion of meaningful rules containing Computer-Efficacy and an overall 10\% difference between portions of meaningful rules containing School Engagement. This suggests strong relationships between ICT engagement, computer-efficacy and school engagement. Results suggest a stronger relationship between ICT Engagement and Computer-Efficacy when students have positive engagement, and one between School Engagement and ICT Engagement when school engagement is negative.

Given the aim of this discussion is to look at variations and differences in students' experiences, the next section takes a more in-depth look at patterns among Computer-Efficacy, School Engagement and ICT Engagement. Selection of these factors for further analysis was based on the most contrast among these factors in the two datasets. Rules containing ComputerEfficacy, School Engagement and ICT Engagement from the two datasets were generated and graphed using fuzzy representations. To generate fuzzy representations, ICT Engagement was calculated on students' Neutral, Positive and Negative beliefs and further calculated to be Low, Medium or High. Computer-Efficacy was calculated on the three sub-factors (see Table 1): Productivity (e.g. email, editing a document), Processing (e.g. creating a graph) and Creating (e.g. making a webpage). Scores were calculated as No Knowledge, Low, Medium and High efficacy. For example, Computer-Efficacy Productivity No Knowledge represents students who 
would have selected "I don't know what this means" (No Knowledge) on most of the productivity items. A Low label represents students who understood most of the tasks, but needed help to perform them. School Engagement was calculated on Neutral, Positive or Negative beliefs. Full reporting of fuzzy representation frequencies is presented in Appendix B. Distributions of rules and graphing of rule distributions are presented in Supplemental B.

Figures 2 and 3 present directed graphs visualizing results from each of the datasets. The association between factors is represented as an arrow connecting two nodes. A node at the beginning of an arrow is an antecedent in the rule, while the end of the arrow is a consequent. Each node has a fuzzy representation label (see Appendix $\underline{B}$ for full list), which may include one or two factors. For example a two factor node would be "SE=negative, $E C=N$ ", where School Engagement Negative and Computer-Efficacy Creating No Knowledge, respectively, where both are part of the antecedent. A one-directional arrow indicates the antecedent factor is likely to have an effect on the consequent factor. A bi-directional arrow shows possible interactive effects between two factors. Arrows with darker lines represent stronger rules with a higher lift. In the following two figures, clusters of factors have been identified. Clusters observed in each dataset are shaded and labeled for discussion purposes.

Figure 2 presents important rules relating to positive ICT engagement (Dataset 1). $<<$ Insert Figure 2>>

Figure 2 presents rules graphed from Dataset 1 and shows two centralized clusters. Cluster 1 reveals a pattern with Computer-Efficacy Processing Low Knowledge as the consequent of seven rules. Cluster 2 has Computer-Efficacy Creation Low Knowledge at the center and as the consequent of seven rules. The two central nodes were weakly connected 
through two-directional relationships with Computer-Efficacy Productivity Low Knowledge. Rules containing School Engagement were not present, which suggests it was not important in this dataset.

The first interesting pattern in this dataset is that only six factors were important: three computer-efficacy low factors, ICT Engagement Positive, ICT Engagement Positive High and ICT Engagement Negative Low. Many nodes presenting as antecedents, in both clusters, contained a combinations of low negative $(\mathrm{EN}=\mathrm{L})$ and high positive $(\mathrm{EP}=\mathrm{H})$ engagement with ICT. This suggests that, for students reporting positive ICT engagement, efficacy on creating tasks and their level of ICT engagement was likely to affect their perceived efficacy on processing tasks (Cluster 1) and vice versa in Cluster 2. However, their level of ICT engagement did not seem to have an effect on perceptions of Computer-Efficacy Productivity Low (EPD=L), which includes less complex tasks. The second interesting pattern in this dataset was that a direct association between Computer-Efficacy Creation Low (EC=L) and Computer-Efficacy Processing Low $(\mathrm{EPC}=\mathrm{L})$ was not present. All rules included antecedents comprising both computer-efficacy and engagement factors. This suggests ICT engagement was very important in this group. An additional interesting aspect of this dataset was that School Engagement was not important. Students having positive feelings about using technology were unlikely to also feel negatively about school.

Figure 3 presents important rules for negative ICT engagement (Dataset 2). $<<$ Insert Figure 3>>

Three clusters were observed in Figure 3. Cluster 1 showed a distributed organization comprising computer-efficacy No Knowledge factors (e.g. EPD=N, Computer-Efficacy 
Productivity No Knowledge). Cluster 2 presented a more centralized pattern and comprises both computer-efficacy and ICT engagement factors, with School Engagement Negative ( $\mathrm{SE}=$ negative) at the center as a consequent for six rules. School Engagement Negative was also in a bi-directional association with medium negative ICT Engagement Medium Negative $(\mathrm{EN}=\mathrm{M})$. Cluster 3 demonstrated a weak centralized pattern with ICT Engagement Negative Medium at the center and as a consequent of four rules. However, the mixture of ICT Engagement Medium Positive (e.g. EP = M) and Medium Negative (e.g. EN=M) and School Engagement Negative factors confirms that even though all students in this dataset reported negative ICT Engagement, there was still a range of beliefs. Unlike Dataset 1, computer-efficacy Low factors were not present.

There are two interesting patterns in this dataset. The first is the importance of School Engagement Negative (SE=negative) in this dataset. Students, who were likely to report medium negative ICT engagement and/or no knowledge on computer tasks, were also likely to feel negatively about school. While the associations were not strong, the centrality of School Engagement Negative as a consequent suggest computer-efficacy and engagement factors were likely to contribute to students' negative beliefs about school. The second interesting pattern is the complexity and distributed nature of associations among the factors, in comparison with the centralized organization of clusters in the positive ICT engagement dataset. This suggests that students feeling negatively about using ICTs have a much more complex experience in technologically integrated learning than those feeling positive about ICTs.

\section{Discussion}

This study aimed to understand variations in students' confidence and engagement with digital technologies in learning and consider possible implications for teachers' learning design. 
To do this, data mining approaches, association rules and fuzzy representations, were used to explore a student questionnaire dataset from a large Australian school one-to-one laptop program. To address the research question: "What are different patterns occurring among key factors relating to students' experiences in technology integration?" results confirm distinctly different patterns among students depending on their ICT engagement. For students reporting positive ICT engagement, the most important factors were Computer-Efficacy Processing and Creating. Other factors of ICT integration related to these two formed a centralized and linear pattern. For students reporting negative ICT engagement, medium negative ICT engagement and negative school engagement were the most important factors. In this group, relationships with other factors were very distributed and without a clear centre, which suggests multiple effects and complex relationships among factors.

This analysis revealed two important differences between the groups: 1) different factors were important; and, 2) associated factors clustered in different ways. These variations can inform how the two groups of students may experience technology integration differently in learning. For students reporting positive ICT engagement the most important factors were Computer-Efficacy Processing Low and Computer-Efficacy Creating Low, both of which demonstrated multiple strong associations with positive engagement. However, the ComputerEfficacy Productivity, Low factor was not associated with engagement, only with the other two computer-efficacy factors. This finding reflects what is often assumed in technology integration, that higher engagement with ICT is related to motivation and confidence performing more creative and complex tasks (see Christoph, Goldhammer, Zylka \& Hartig, 2014; Liard \& Kuh, 2005). In regard to technologically integrated learning designs and classroom tasks, students who are positively engaged with technology use could be reasonably challenged with more complex 
learning tasks using digital technologies. For example, students could be given inquiry or problem-based tasks requiring them to select different ICTs to research, process information and visually present a solution. These students would be likely to feel confident they could complete more complicated tasks successfully. More complex ICT use is thought to lead to stronger engagement in learning and higher order thinking skills (Silva, 2009). Simplistic productivity tasks were not associated with engagement and were less important for this group. However, tasks must still be appropriately designed and novel aspects clearly presented or even confident students can quickly become frustrated (Tzeng, 2009).

However, findings suggest a very different experience for students reporting negative engagement in ICT. In the negative ICT engagement group, students felt less confident performing computer-related tasks, which was shown by Computer-Efficacy No Knowledge factors being more important in this group. In particular, Productivity exhibited strong and more frequent associations with other factors, including School Engagement Negative and ICT Engagement Negative Medium. However, a linear relationship did not exist among these three important factors, which suggests complex and indirect effects from each factor. The connectedness of these factors suggests students' low confidence performing productivity tasks is likely to have a unidirectional and direct effect on negative school engagement and a combined effect with negative ICT engagement. While associations between lower computerefficacy and negative ICT engagement are not surprising, their effect on negative school engagement is a key finding. The complexity of this pattern has implications for the belief that integrating ICTs in tasks can engage lower performing students in learning (e.g. Perrotta, 2013; Windschitl \& Sahl, 2002). Findings suggest that negative feelings about using technologies or tasks that are too challenging in learning may further contribute to negative school engagement. 
Yet, while a student may not be interesting in digital technologies, having and knowing about technology is a key part of contemporary education. For example, a students struggling to perform basic computer tasks, such as email or Internet searching, may feel ashamed and resent the learning task. We propose that to engage these students in learning and school, through the use of ICTs and technologically integrated learning, that positive experiences to increase confidence with computers should be part of learning designs. By acknowledging the complexity of integration factors for students who are less engaged and less confident using ICTs, learning design can become more responsive to their needs. Importantly, analysis suggests that computerefficacy factors are more important than engagement in this group and have a stronger effect on other factors. This can help teachers identify where to focus time and resources in learning design and in the classroom.

Thus, an alternative to the prior example would be to pair students together who have complimentary computer knowledge and scaffold less confident students' work to build their knowledge of digital technologies. Teachers may then scaffold increasing complexity of technology skills into learning designs, to building students up to more sophisticated computer use, such creating graphs or webpages. Students should not be introduced to more complex practice until they have mastered and feel confident performing more basic tasks (see Aesaert \& van Braak, 2014). These approaches may become problematic if teachers feel they are teaching technology skills, rather than curricula content. Further, teachers that do not have a high level of confidence using ICTs may further complicate students' experience using digital technologies (see Warschauer et al., 2014).

It is important to note that this analysis only shows associations among factors and not causal relationships. Further, that school and ICT engagement may be functions of students' 
beliefs about their learning success or other experiences in school. Moreover, different students may struggle or become disengaged with a task for different reasons (Tzeng, 2009). Regardless, it is important that the complexity of some students' experiences with technologically integrated be acknowledged and that technology integration may not result in positive effects on learning. Our research also suggests that variations in students' experiences can be identified, which could then be addressed in learning design.

\section{Future research and conclusions}

While our analysis was quite focused, results already show important variations between student experiences and suggest considerations for teachers' learning designs. The immediate next step in this work will be to validate findings using a second student dataset. To do this we will use the Year 10 student questionnaire dataset collected as part of the NSW-DER study in 2013. Both data collections included Computer-Efficacy, ICT Engagement and School Engagement factors and the five other key factors identified. Validation of associations among factors will allow for future building on these findings, in our own work and work in the field (see Baker \& Yacef, 2009).

The second step of development will be to widen the analysis and explore associations including other key factors from the conceptual framework, particularly students' Learning Preferences, Learning Beliefs and ICT Learning Performance. This will help to further unpack implications of patterns observed among Computer-Efficacy, ICT Engagement and School Engagement and begin to draw stronger connections to learning. We will also consider including other key factors from questionnaire Part B and triangulate with Part A, to explore Access, Gender and Intentions after high school, which have all been shown to influence students' perceptions of digital technology use (e.g. Robinson et al., 2015; Tømte \& Hatlevik, 2011). This 
would result in a more comprehensive model of students' experiences in technologically integrated learning. As part of model development, we will explore the use of association rule summary techniques to automate identification of frequently occurring rules and related sub rules (e.g. Liu, Hsu, \& Ma, 1999) to increase efficiency and accuracy of identifying important rules. Student and teacher datasets, particularly on Teacher Directed Use of ICT and ICT Importance in Subject Areas, will also be compared to investigate if they have different perspectives and understanding of the technologically integrated learning designs and experiences (Könings, Seidel, \& van Merriënboer, 2014).

There were a few key limitation of this analysis. First, the algorithm chosen was apriori, which is widely used for association rule mining, but it is strongly affected by sample size and parameter settings. Therefore, important rules occurring in smaller subsets of the data can be obscured. While on one hand only the most frequent and strongest rules are identified, this can limit exploration of variations among rules. Second, this would also be affected by distributions and trends occurring in the student dataset. Generation of fuzzy representations and association rules analysis resulted in the majority of important rules having antecedent and consequence labels containing Negative or Low. This may result from skewness in the data, which can affect how fuzzy representations are defined. The questionnaire dataset contained many responses from students, which were incomplete, meaningless or missing. Some of these issues were treated in data processing stages, but not all problems can be corrected or records removed. This cleaning process may affect accuracy of the dataset. To account for these limitations, future research will split the dataset and test additional processes, such as refining and combining different aspects of the data and scales to more accurately explore variation within the sample. Furthermore, for simplicity we did not calculate the graph weighting in the visualization while representing 
generated rules as the directed graph. Nevertheless, the weights are usually associated with the importance of the connections among different factors. Therefore, another avenue of future work is to identify the graph weighting for generated rules.

With this said, results suggest important variations in students' experiences in technologically integrated learning. A key finding of this analysis was that technologically integrated learning may be more complex for students holding negative ICT engagement. Ultimately, students' engagement in ICTs cannot be assumed and, in some cases, may in fact effect engagement in school and learning. Teachers need to be careful to address issues of ICT engagement and efficacy in learning designs, to insure all students are able to participate in and benefit from technologically integrated learning.

\section{Acknowledgements}

$<<$ insert Acknowledgements here $>>$

\section{References}

Author/s. (2011). Removed for peer review.

Author/s. (2013). Removed for peer review.

Author/s. (2015). Removed for peer review.

Australian Bureau of Statistics. (2016). Table 40a number of full-time students, 2001-2015. Canberra. Retrieved from http://www.abs.gov.au/.

Australian Council for Educational Research. (2009). School life questionnaire. Camberwell, VIC.

Amemiya, Y., \& Anderson, T. W. (1990). Asymptotic chi-square tests for a large class of factor analysis models. The Annals of Statistics, 18(3), 1453-1463. doi:10.1214/aos/1176347760

Aesaert, K., \& van Braak, J. (2014). Exploring factors related to primary school pupils' ICT selfefficacy: A multilevel approach. Computers in Human Behavior, 41, 327-341. doi:10.1016/j.chb.2014.10.006 
Baker, R. S. J. D. (2010). Data mining for education. In International Encyclopaedia of Education (pp. 112-118). Elsevier. doi.org/10.1016/B978-0-08-044894-7.01318-X

Baker, R. S. J. D., \& Yacef, K. (2009). The state of educational data mining in 2009: A review and future visions. Journal of Educational Data Mining, 1(1), 3-17.

Baylor, A. L., \& Ritchie, D. (2002). What factors facilitate teacher skill, teacher moral, and perceived student learning in technology-using classrooms? Computers \& Education, 39, 395-414. doi:10.1016/S0360-1315(02)00075-1

Bebell, D., \& Kay, R. (2010). One to one computing: A summary of the quantitative results from the Berkshire Wireless Learning Initiative. The Journal of Technology, Learning and Assessment, 9(2), 5-58.

Bebell, D., \& O'Dwyer, L. M. (2010). Educational outcomes and research from 1:1 computing settings. Journal of Technology, Learning, and Assessment, 9(1), 4-14.

Beck, J. E., Chang, K. M., Mostow, J., \& Corbett, A. (2008). Does help help? Introducing the Bayesian Evaluation and Assessment methodology. In Intelligent tutoring systems (pp. 383394). Berlin Heidelberg: Springer.

Becker, H. J. (1994). How exemplary computer-using teachers differ from other teachers: Implications... Journal of Research on Computing in Education, 26(3), 291. Retrieved from http://search.ebscohost.com/login.aspx?direct=true \&db=a9h\&AN=9502070435\&site=ehost -live

Bennett, S., \& Maton, K. (2010). Beyond the "digital natives" debate: Towards a more nuanced understanding of students' technology experiences. Journal of Computer Assisted Learning, 26(5), 321-331. doi:10.1111/j.1365-2729.2010.00360.x

Breiman, L. (2001). Statistical modeling: The two cultures. Statistical Science, 16(3), 199-231.

Chen, G., \& Wei, Q. (2002). Fuzzy association rules and the extended mining algorithms. Information Sciences, 147(1-4), 201-228. doi:10.1016/S0020-0255(02)00264-5

Chen, Y.-L., \& Weng, C.-H. (2009). Mining fuzzy association rules from questionnaire data. Knowledge-Based Systems, 22(1), 46-56. doi:10.1016/j.knosys.2008.06.003

Christoph, G., Goldhammer, F., Zylka, J., \& Hartig, J. (2015). Adolescents' computer performance: The role of self-concept and motivational aspects. Computers \& Education, 81, 1-12. http://doi.org/10.1016/j.compedu.2014.09.004

Compeau, D. R., \& Higgins, C. A. (1995). Computer self-efficacy: Development of a measure and initial test. MIS Quarterly, 19(2), 189-211.

Department of Education and Training. (2002). SchoolMap best practices statements. Sydney. 
Department of Education Employment and Workplace Relations. (2012). Digital Education

Revolution - Overview. Digital Education Revolution - Overview. Retrieved September 20, 2013, from

http://www.deewr.gov.au/Schooling/DigitalEducationRevolution/Pages/default.aspx

Ertmer, P. A., \& Ottenbreit-Leftwich, A. T. (2010). Teacher technology change: How knowledge, confidence, beliefs, and culture intersect. Journal of Research on Technology in Education, 42(3), 255-284. doi:GALEIA221849729

Fairlie, R. W., \& London, R. A. (2011). The effects of home computers on educational outcomes: Evidence from a field experiment with community college students. The Economic Journal, 122(561), 727-753. doi:10.1111/j.1468-0297.2011.02484.x.

Fayyad, U., Piatestsky-Sharpiro, G., \& Smyth, P. (1996). From data mining to knowledge discovery in databases. AI Magazine, 17(3), 37-54.

Gibson, P. A., Stringer, K., Cotten, S. R., Simoni, Z., O’Neal, L. J., \& Howell-Moroney, M. (2014). Changing teachers, changing students? The impact of a teacher-focused intervention on students' computer usage, attitudes, and anxiety. Computers \& Education, 71, 165-174. doi:10.1016/j.compedu.2013.10.002

Han, J., Kamber, M., \& Pei, J. (2012). Data mining: Concepts and techniques. 3rd Edition, Morgan Kaufmann Publisher.

Harris, J., Mishra, P., \& Koehler, M. (2009). Teachers' technological pedagogical content knowledge and learning activity types. Journal of Research on Technology in Education, 41(4), 393-416. http://doi.org/10.1080/15391523.2009.10782536

Hasan, B. (2003). The influence of specific computer experiences on computer self-efficacy beliefs. Computers in Human Behavior, 19(4), 443-450. doi:10.1016/s07475632(02)00079-1

Hatlevik, O. E., Guðmundsdóttir, G. B., \& Loi, M. (2014). Digital diversity among upper secondary students: A multilevel analysis of the relationship between cultural capital, selfefficacy, strategic use of information and digital competence. Computers \& Education, 81, 345-353. doi:10.1016/j.compedu.2014.10.019

Hofer, B. K., \& Pintrich, P. R. (1997). The development of epistemological theories: Beliefs about knowledge and knowing and their relation to learning. Review of Educational Research, 67(1), 88-140. Retrieved from http://www.jstor.org/stable/1170620

Inan, F. A., \& Lowther, D. L. (2010). Laptops in the K-12 classrooms: Exploring factors impacting instructional use. Computers \& Education, 55(3), 937-944. doi:10.1016/j.compedu.2010.04.004 
Kim, C., Kim, M. K., Lee, C., Spector, J. M., \& DeMeester, K. (2013). Teacher beliefs and technology integration. Teaching and Teacher Education, 29(0), 76-85.

doi:http://dx.doi.org/10.1016/j.tate.2012.08.005

Klir, G.J. \& Yuan, B. (1995). Fuzzy sets and fuzzy logic: Theory and applications. Prentice Hall.

Könings, K., Seidel, T., Brand-Gruwel, S., \& van Merriënboer, J. G. (2014). Differences between students' and teachers' perceptions of education: Profiles to describe congruence and friction. Instructional Science, 42(1), 11-30. doi:10.1007/s11251-013-9294-1

Könings, K., Seidel, T., \& van Merriënboer, J. G. (2014). Participatory design of learning environments: Integrating perspectives of students, teachers, and designers. Instructional Science, 42(1), 1-9. doi:10.1007/s11251-013-9305-2

Kozma, R. B. (2003). Technology and Classroom Practices. Journal of Research on Technology in Education, 36(1), 1-14. http://doi.org/10.1080/15391523.2003.10782399

Laird, T. N., \& Kuh, G. (2005). Student experiences with information technology and their relationship to other aspects of student engagement. Research in Higher Education, 46(2), 211-233. http://doi.org/10.1007/s11162-004-1600-y

Lamont, A., \& Maton, K. (2010). Unpopular music: Beliefs and behaviours towards music in education. In R. Wright (Ed.), Sociology and Music Education (pp. 63-80). London: Ashgate.

Lei, J., \& Zhao, Y. (2007). Technology uses and student achievement: A longitudinal study. Computers \& Education, 49(2), 284-296. doi:10.1016/j.compedu.2005.06.013

Li, Q. (2007). Student and teacher views about technology. Journal of Research on Technology in Education, 39(4), 377-397. doi:10.1080/15391523.2007.10782488

Liu, B., Hsu, W., \& Ma, Y. (1999). Pruning and summarizing the discovered associations. In Proceedings of the Fifth ACM SIGKDD International Conference on Knowledge Discovery (pp. 125-134). doi:10.1145/312129.312216

Lowther, D. L., Inan, F. A., Ross, S. M., \& Strahl, J. D. (2012). Do one-to-one initiatives bridge the way to 21st century knowledge and skills? Journal of Educational Computing Research, 46(1), 1-30. doi:10.2190/EC.46.1.a

Margaryan, A., Littlejohn, A., \& Vojt, G. (2011). Are digital natives a myth or reality? University students' use of digital technologies. Computers \& Education, 56(2), 429-440. doi:10.1016/j.compedu.2010.09.004

Merceron, A., \& Yacef, K. (2010). Measuring correlation of strong symmetric assocation rules in educational data. In C. Romero, S. Ventura, M. Pechenizkiy, \& R. S. J. D. Baker (Eds.), 
Handbook of Educational Data Mining (pp. 245-255). Boca Raton: Taylor \& Francis Group.

Moos, D. C., \& Azevedo, R. (2009). Learning with computer-based learning environments: A literature review of computer self-efficacy. Review of Educational Research, 79(2), 576600. doi:10.3102/0034654308326083

Muis, K., \& Duffy, M. (2013). Epistemic climate and epistemic change: Instruction designed to change students' beliefs and learning strategies and improve achievement. Journal of Educational Psychology, 105(1), 213-225. doi:10.1037/a0029690

Mumtaz, S. (2000). Factors affecting teachers' use of information and communications technology: A review of the literature. Technology, Pedagogy and Education, 9(3), 319342. doi:10.1080/14759390000200096

OECD. (2006). PISA 2006 Information and communication technology questionnaire. OECD Program for International Student Assessment.

Ottenbreit-Leftwich, A. T., Glazewski, K. D., Newby, T. J., \& Ertmer, P. A. (2010). Teacher value beliefs associated with using technology: Addressing professional and student needs. Computers \& Education, 55(3), 1321-1335. doi:http://dx.doi.org/10.1016/j.compedu.2010.06.002

Newman, M. (2003). The structure and function of complex networks. SIAM Review, 45(2), 167-256. doi:10.1137/S003614450342480

Pellas, N. (2014). The influence of computer self-efficacy, metacognitive self-regulation and self-esteem on student engagement in online learning programs: Evidence from the virtual world of Second Life. Computers in Human Behavior, 35, 157-170. doi:10.1016/j.chb.2014.02.048

Perrotta, C. (2013). Do school-level factors influence the educational benefits of digital technology? A critical analysis of teachers' perceptions. British Journal of Educational Technology, 44(2), 314-327. doi:10.1111/j.1467-8535.2012.01304.x

Philip, T., \& Garcia, A. (2013). The importance of still teaching the iGeneration: New technologies and the centrality of pedagogy. Harvard Educational Review, 83(2), 300-319. doi.org/10.17763/haer.83.2.w221368g1554u158

Reyes, M. R., Brackett, M. A., Rivers, S. E., White, M., \& Salovey, P. (2012). Classroom emotional climate, student engagement, and academic achievement. Journal of Educational Psychology, 104(3), 700-712. doi.org/10.1037/a0027268

Robinson, L., Cotten, S. R., Ono, H., Quan-Haase, A., Mesch, G., Chen, W., ... Stern, M. J. (2015). Digital inequalities and why they matter. Information, Communication \& Society, 18(5), 569-582. doi.org/10.1080/1369118X.2015.1012532 
Roehrig, G. H., Kruse, R. A., \& Kern, A. (2007). Teacher and school characteristics and their influence on curriculum implementation. Journal of Research on Science Teaching, 44(7), 883-907.

Selwyn, N. (2009). The digital native - myth and reality. Aslib Proceedings, 61(4), 364-379.

Shi, J., \& Malik, J. (2000). Normalized cuts and image segmentation, IEEE Transactions On Pattern Analysis And Machine Intelligence. 22(8): 888-905. doi: 10.1109/34.868688

Silva, E. (2009). Measuring for 21st century learning. The Phi Delta Kappan, 90(9), 630-634. http://www.jstor.org/stable/27652741

Skryabin, M., Zhang, J., Liu, L., \& Zhang, D. (2015). How the ICT development level and usage influence student achievement in reading, mathematics, and science. Computers \& Education, 85, 49-58. doi:10.1016/j.compedu.2015.02.004

Stephenson, K., \& Zelen, M. (1989). Rethinking centrality: Methods and examples. Social Networks, 11(1), 1-37. doi:10.1016/0378-8733(89)90016-6

Tan, P.N., Steinbach, M., \& Kumar, V. (2005) Introduction to data mining ( $1^{\text {st }}$ Edition), Addison-Wesley.

Tamim, R. M., Bernard, R. M., Borokhovski, E., Abrami, P. C., \& Schmid, R. F. (2011). What forty years of research says about the impact of technology on learning. Review of Educational Research, 81(1), 4-28. doi:10.3102/0034654310393361

Thompson, P. (2013). The digital natives as learners: Technology use patterns and approaches to learning. Computers \& Education, 65, 12-33.

doi:http://dx.doi.org/10.1016/j.compedu.2012.12.022

Tømte, C., \& Hatlevik, O. E. (2011). Gender-differences in self-efficacy ICT related to various ICT-user profiles in Finland and Norway. How do self-efficacy, gender and ICT-user profiles relate to findings from PISA 2006. Computers \& Education, 57(1), 1416-1424. doi.org/10.1016/j.compedu.2010.12.011

Trigwell, K., \& Prosser, M. (2004). Development and use of the approaches to teaching inventory. Educational Psychology Review, 16(4), 409-424. doi:10.1007/s10648-004-00079

Tzeng, J.-Y. (2009). The impact of general and specific performance and self-efficacy on learning with computer-based concept mapping. Computers in Human Behavior, 25(4), 989-996. doi:10.1016/j.chb.2009.04.009

Vermunt, J. D., \& Verloop, N. (1999). Congruence and friction between learning and teaching. Learning and Instruction, 9(3), 257-280. doi:10.1016/S0959-4752(98)00028-0 
Wang, S.-K., Hsu, H.-Y., Campbell, T., Coster, D., \& Longhurst, M. (2014). An investigation of middle school science teachers and students use of technology inside and outside of classrooms: considering whether digital natives are more technology savvy than their teachers. Educational Technology Research and Development, 62(6), 637-662. doi:10.1007/s11423-014-9355-4

Warschauer, M., \& Matuchniak, T. (2010). New technology and digital worlds: Analyzing evidence of equity in access, use, and outcomes. Review of Research in Education, 34(1), 179-225. doi:10.3102/0091732x09349791

Warschauer, M., Zheng, B., Niiya, M., Cotten, S., \& Farkas, G. (2014). Balancing the one-to-one equation: Equity and access in three laptop programs. Equity \& Excellence in Education, 47(1), 46-62. doi:10.1080/10665684.2014.866871

Waycott, J., Bennett, S., Kennedy, G., Dalgarno, B., \& Gray, K. (2010). Digital divides? Student and staff perceptions of information and communication technologies. Computers \& Education, 54(4), 1202-1211. doi:http://dx.doi.org/10.1016/j.compedu.2009.11.006

Yu, K., Yu, S., \& Tresp, V. (2005). Soft clustering on graphs. In Advances in neural information processing systems, 18 (pp. 1553-1560). 